\title{
Ultrasensitive detection of bacteria using core-shell nanoparticles and a NMR-filter system ${ }^{* *}$
}

\author{
Hakho Lee ${ }^{1,+}$, Tae-Jong Yoon ${ }^{1,+}$, and Ralph Weissleder ${ }^{1,2}$ \\ Ralph Weissleder: rweissleder@mgh.harvard.edu \\ ${ }^{1}$ Center for Systems Biology, Massachusetts General Hospital / Harvard Medical School, 185 \\ Cambridge Street, Boston, MA 02114 (USA) \\ ${ }^{2}$ Department of Systems Biology, Harvard Medical School, 200 Longwood Av., Boston, MA 02115 \\ (USA), Fax: (+1) 617-643-6133
}

\section{Keywords}

nanoparticles; biosensors; nanotechnology; NMR; microfluidics

\begin{abstract}
Direct detection of pathogens is key in combating human infections, in identifying nosocomial sources, in surveying food chains and in biodefense.[1] Recent advances in nanotechnology have enabled the development of new diagnostic platforms[2] aimed at more sensitive and faster pathogen detection.[3] Many of the reported technologies, albeit elegant, often fail in routine clinical settings[4] because they still require extensive specimen purification, use complex measurement setups, or are not easily scalable for clinical demands. Here we report a new, simple, nanoparticle-based platform that can rapidly detect pathogens in native biological samples. In this approach, bacteria are targeted by highly magnetic nanoparticles (MNP), concentrated into a microfluidic chamber, and detected by nuclear magnetic resonance (NMR). The clinical utility of our diagnostic platform was evaluated by detecting tuberculosis (TB), a leading cause of disease and death worldwide.[5] Using the bacillus Calmette-Guérin (BCG) as a surrogate for Mycobacterium tuberculosis, we demonstrate unprecedented detection speed and sensitivity; as few as 20 colony-forming unit (CFU) in sputum $(1 \mathrm{~mL})$ were detected in $<30 \mathrm{~min}$. With the capability for fast, simple and portable operation, the new detection platform could be an ideal point-of-care diagnostic tool, especially in resource-limited settings.
\end{abstract}

The diagnosis starts with specimen collection and incubation with bacteria specific MNP (Supporting Information Figure S1). MNP bind to the bacterial wall, rendering the bacteria superparamagnetic. In a subsequent step the spin-spin relaxation time $\left(T_{2}\right)$ of the whole sample is measured by NMR. As the magnetic fields from MNP dephase the precession of nuclear spins in water protons[6, 7], each MNP-tagged bacterium can shorten the $T_{2}$ of billions of surrounding water molecules. To increase detection sensitivity, we have incorporated signal amplification schemes that made it possible to detect small quantities of bacteria in relatively large sample volumes. At the nanoparticle level, the detection signal has been enhanced by synthesizing Fe-based MNP with the high transverse relaxivity $\left(r_{2}\right)$.

\footnotetext{
** The authors thank D. Hung (MGH) and M. Cima (MIT) for many helpful discussions; R.M. Westervelt (Harvard) for support in device fabrication; N. Sergeyev (MGH) for synthesizing CLIO.

Correspondence to: Ralph Weissleder, rweiss leder@mgh. harvard. edu.

${ }^{+}$These authors contributed equally.

Supporting information for this article is available on the WWW under http://www.angewandte.org or from the author.
} 
At the device level, the signal was amplified by concentrating bacteria into a microfluidic chamber where the NMR signal was measured. To provide portable, on-chip bacterial detection, the NMR signal was read out using a miniaturized NMR system we have recently developed.[8].

First, we developed hybrid MNP with a large Fe core and a thin ferrite shell ("cannonballs"; $\mathrm{CB}$ ), that have very high $r_{2}$ per particle (Table S1). With the limited number of binding sites per bacterium, the $T_{2}$ of samples will be shorter and thereby the detection will be more sensitive when the individual MNP have higher $r_{2}$. Since $r_{2}$ is $\propto M^{2} \bullet d^{2}$, where $M$ and $d$ are the particle magnetization and the diameter respectively[6], we focused on making larger MNP using highly magnetic material $(\mathrm{Fe})$. Figure 1a shows an example of highly monodisperse CB $(d=16 \mathrm{~nm})$. Initially, we made Fe-only MNP by thermally decomposing $\mathrm{Fe}(\mathrm{CO})_{5}$, followed by controlled air-oxidation to grow the ferrite shell.[9] Compared to chemical oxidation[10], our method produces a thinner shell and thereby leaves a larger $\mathrm{Fe}$ core (Figure S2), leading to higher $M$. The shell showed high crystallinity (Figure 1b) and $\mathrm{X}$-ray diffraction revealed a typical pattern for a spinel structure (Figure 1c), confirming the ferrite nature of the shell.[11] The shell protected the Fe core from oxidation to maintain the magnetic properties of CB (Figure S3). CB showed high magnetization (139 emu g ${ }^{-1}[\mathrm{Fe}]$ ) and yet were superparamagnetic at room temperature (Figure 1d). Most importantly, CB assumed high $r_{2}\left(=6.1 \times 10^{-11} \mathrm{~m} \mathrm{~L} \mathrm{~s}^{-1}, 1.5 \mathrm{~T}\right.$; Figure $\left.\mathrm{S} 4\right)$, due to their high magnetization and large diameter.

To render $\mathrm{CB}$ specific for BCG, we conjugated anti-BCG monoclonal antibodies to their surface (CB-BCG; see Methods in Supporting Information). Bacterial samples were then incubated with CB-BCG for $10 \mathrm{~min}$, followed by a wash step to remove excess particles. Optical microscopy with fluorescent CB-BCG showed excellent targeting (Figure 2a). Binding of CB-BCG was further verified by the element mapping (Figure 2b), which showed high Fe signal on the bacterial membrane. The number of CB-BCG per bacterium, quantified by inductively coupled plasma atomic emission spectroscopy (ICP-AES; Figure S5), was $\approx 10^{5}$. Magnetic tagging thus made the bacteria highly efficient $T_{2}$-shortening agents (Figure 2c). The binding of CB-BCG to BCG could be inhibited by antibody blockade against bacterial epitopes. For control CB or when CB-BCG were used against other bacterial strains, the CB binding was minimal with $T_{2}$ changes $\left(\Delta T_{2}\right)<5 \%$. The number of CB-BCG tested against different bacteria species were $<600$ per bacterium (Figure S5), confirming the specificity of CB-BCG.

To further enhance detection sensitivity and to streamline assay procedures, we next developed a chip-based filter system with NMR compatibility (Figure 3). A key component is a microfluidic chamber enclosed by a membrane filter and surrounded by a microcoil. The membrane filter performs two functions. First, it captures bacteria and concentrates them into the microfluidic chamber for NMR detection, providing a way to detect a small number of bacteria from large sample volumes. Second, the filter enables on-chip separation of bacteria from unbound $\mathrm{CB}$, obviating the need for separate off-chip purification steps. Figure 4a illustrates the operating principle of the NMR-filter system. An unpurified sample is introduced into the microfluidic channel. Bacteria are retained by the membrane (pore size $\approx 100 \mathrm{~nm}$ ), while excess CB permeate. Subsequently, a buffer solution is repeatedly injected to wash off unbound $\mathrm{CB}$ and the membrane is backwashed to redisperse the captured bacteria. Figure $4 \mathrm{~b}$ shows an operation example with a sample containing BCG and CBBCG. After sample loading, the microfluidic channel was flushed with PBS to remove unbound CB-BCG. Finally, the flow direction was reversed to resuspend the captured BCG for NMR measurements. The number of washing steps to remove unbound CB-BCG was determined by measuring $T_{2}$ after each wash (Figure $4 \mathrm{c}$ ). $T_{2}$ values plateaued in 4-5 washing steps with the whole washing procedure complete in $<5 \mathrm{~min}$. The electron 
micrograph of the membrane after complete wash (Figure 4d) verified bacterial capture on the filter. Note that by removing unbound particles, CB-induced $T_{2}$ changes are much more pronounced than the previously described homogeneous assay[8], achieving $>10^{3}$ times higher detection sensitivity.

To compare the effect of MNP relaxivity on detection sensitivity, we first used a NMR system without a membrane filter. Two types of nanoparticles, CB and cross-linked iron oxide[12] (CLIO; $r_{2}=7.0 \times 10^{-13} \mathrm{~mL} \mathrm{~s}^{-1}$ ), were used (Figure 5, black and blue curves). CBBCG showed much higher mass sensitivity, detecting as few as $6 \mathrm{CFU}$ (in $1 \mu \mathrm{L}$ sample volume), whereas the detection limit was $\sim 100 \mathrm{CFU}$ with CLIO-BCG. Using the NMR-filter system, we achieved substantially high concentration detection sensitivity (Figure 5, red curve). When BCG samples (100 $\mu \mathrm{L})$ targeted with CB-BCG were filtered, the

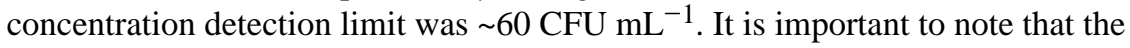
concentration limit is theoretically unlimited with the filtering, because bacteria can be concentrated into the NMR detection chamber from large sample volumes.

To evaluate the clinical utility of the NMR-filter system, we performed comparative detection assays (Table S3). Pulmonary samples were prepared by spiking BCG into human sputa. Following liquefaction, samples were subjected to standard TB diagnostic tests, culture and acid-fast bacilli (AFB) smear microscopy, and CB-based NMR measurements. Without filtration, NMR measurements had a similar sensitivity to AFB smear microscopy, with a detection threshold of $\sim 10^{3} \mathrm{CFU} / \mathrm{mL}$. However, the NMR method was less prone to human error and less labor-intensive. With the NMR-filter system, the detection sensitivity was comparable to that of culture-based detection; when 1-mL of samples were filtered, the detection limit was $\sim 20 \mathrm{CFU}$. The NMR-based detection was much faster $(<30 \mathrm{~min})$ and was performed on a single microfluidic chip, markedly contrasting with the culture-based test that was time-consuming (> 2 weeks) and facility-dependent (e.g. incubators). These results demonstrate that the CB-based NMR-filter system can be readily applied for TB diagnosis in clinical settings.[13]

\section{Experimental Section}

Details on the experimental method are available in Supporting Information online.

\section{Supplementary Material}

Refer to Web version on PubMed Central for supplementary material.

\section{References}

1. a) Batt C. Science 2007;316:1579. [PubMed: 17569853] b) Enserink M. Science 2001;294:1266. [PubMed: 11701907] c) Struelens M, Denis O, Rodriguez-Villalobos H. Microbes. Infect 2004;6:1043. [PubMed: 15345237]

2. a) Luo P, Stutzenberger F. Adv. Appl. Microbiol 2008;63:145. [PubMed: 18395127] b) Rosi N, Mirkin C. Chem. Rev 2005;105:1547. [PubMed: 15826019]

3. a) Grossman H, Myers W, Vreeland V, Bruehl R, Alper M, Bertozzi C, Clarke J. Proc. Natl. Acad. Sci. USA 2004;101:129. [PubMed: 14688406] b) Phillips R, Miranda O, You C, Rotello V, Bunz U. Angew. Chem. Int. Ed. Engl 2008;47:2590. [PubMed: 18228547] c) Zhao X, Hilliard L, Mechery S, Wang Y, Bagwe R, Jin S, Tan W. Proc. Natl. Acad. Sci. USA 2004;101:15027. [PubMed: 15477593]

4. Urdea M, Penny L, Olmsted S, Giovanni M, Kaspar P, Shepherd A, Wilson P, Dahl C, Buchsbaum S, Moeller G, Hay Burgess D. Nature 2006;444 Suppl 1:73. [PubMed: 17159896]

5. Keeler E, Perkins M, Small P, Hanson C, Reed S, Cunningham J, Aledort J, Hillborne L, Rafael M, Girosi F, Dye C. Nature 2006;444 Suppl 1:49. [PubMed: 17159894] 
6. Brooks R. Magn. Reson. Med 2002;47:388. [PubMed: 11810684]

7. Perez J, Josephson L, O'Loughlin T, Hogemann D, Weissleder R. Nat. Biotechnol 2002;20:816. [PubMed: 12134166]

8. Lee H, Sun E, Ham D, Weissleder R. Nat. Med 2008;14:869. [PubMed: 18607350]

9. Cabot A, Puntes V, Shevchenko E, Yin Y, Balcells L, Marcus M, Hughes S, Alivisatos A. J. Am. Chem. Soc 2007;129:10358. [PubMed: 17676738]

10. Peng S, Wang C, Xie J, Sun S. J. Am. Chem. Soc 2006;128:10676. [PubMed: 16910651]

11. Jiao F, Jumas J, Womes M, Chadwick A, Harrison A, Bruce P. J. Am. Chem. Soc 2006;128:12905. [PubMed: 17002386]

12. Josephson L, Tung CH, Moore A, Weissleder R. Bioconjug. Chem 1999;10:186. [PubMed: 10077466]

13. Perkins M. Int. J. Tuberc. Lung Dis 2000;4:S182. [PubMed: 11144551] 
a)

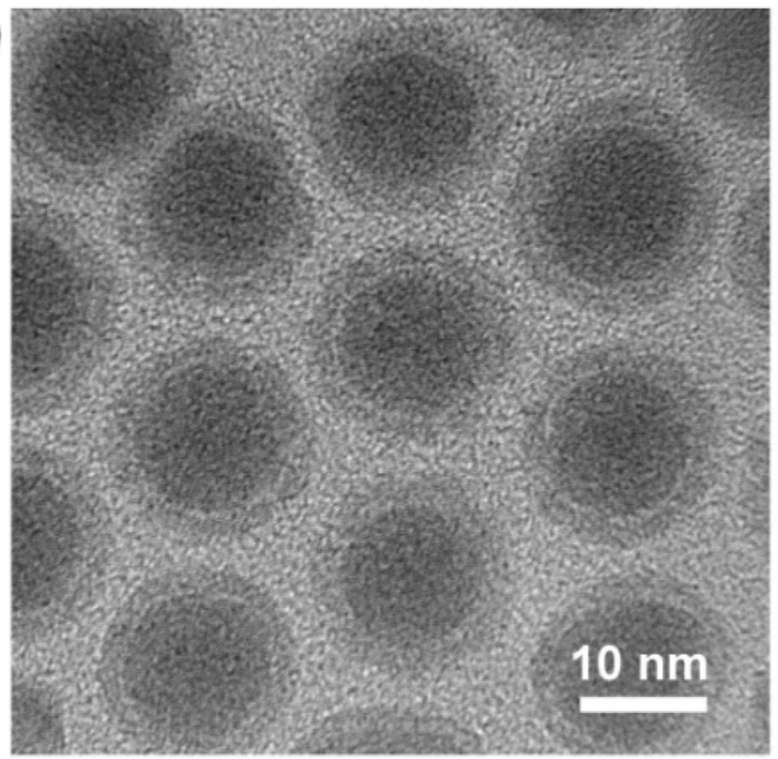

c)

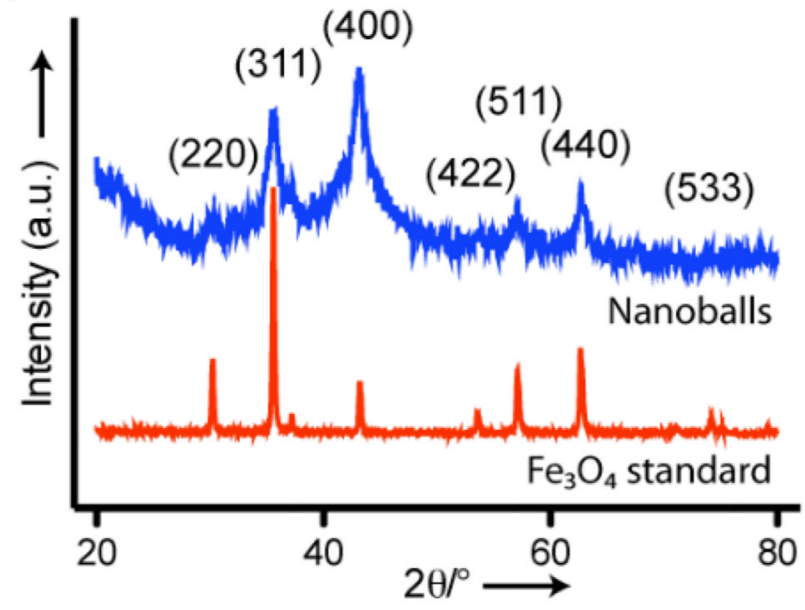

b)

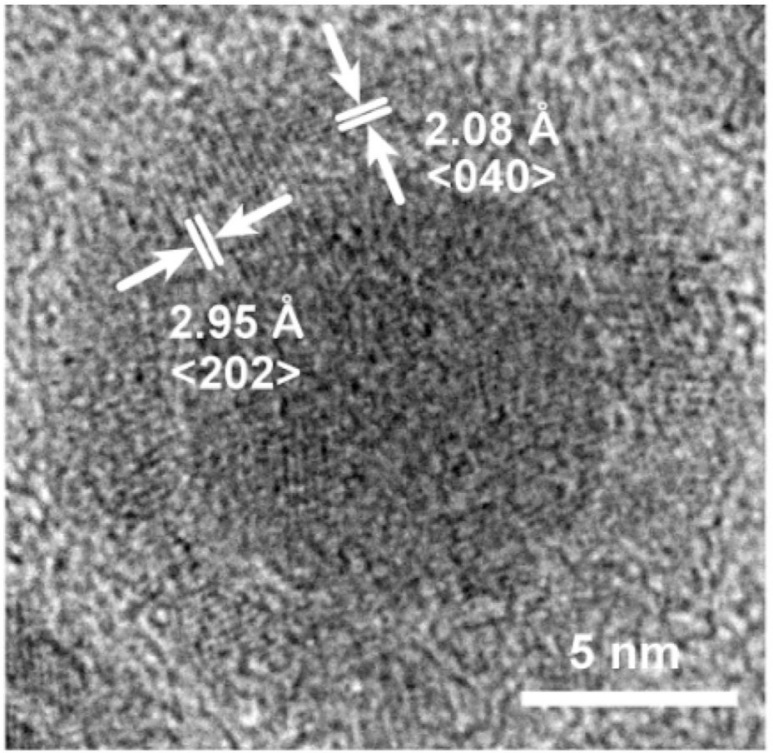

d)

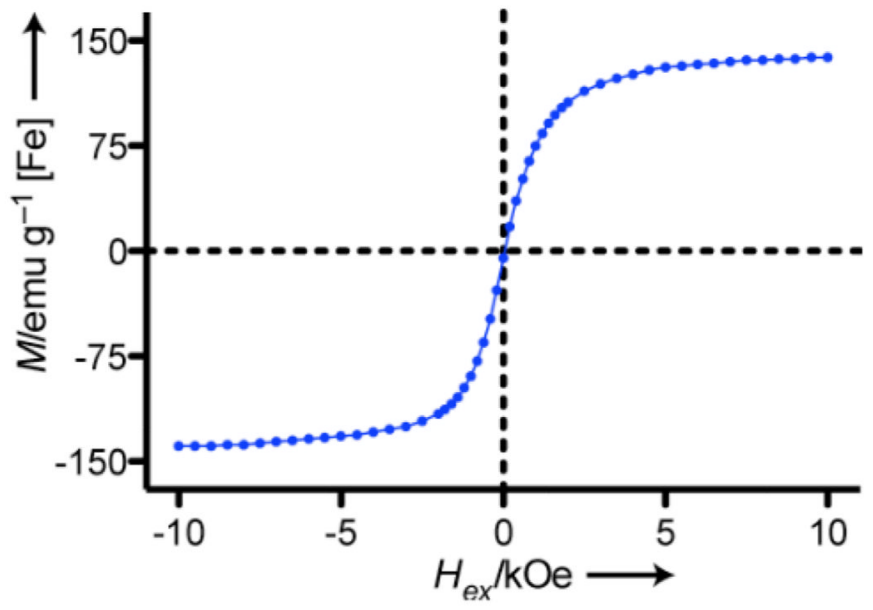

Figure 1.

Cannonballs (CB) for bacterial targeting. a) The particles have a large metallic core $(\mathrm{Fe})$ passivated with a thin ferrite shell, resulting in high particle relaxivity $\left(>5 \times 10^{-11} \mathrm{~mL} \mathrm{~s}^{-1}\right.$ at $0.5 \mathrm{~T}$ ). The core diameter and the shell thickness are 11 and $2.5 \mathrm{~nm}$, respectively. $\mathrm{CB}$ are highly mono-disperse and well-suspended in solution. b) High resolution image of CB showing the crystallinity of the iron-oxide shell with lattice constants. The shell consists of multiple domains of single crystals, due to the lattice mismatch between the iron-oxide shell and $\mathrm{Fe}$ core. $\mathrm{c}$ ) The X-ray powder diffractogram of $\mathrm{CB}$ reveals a typical spinel structure as in $\mathrm{Fe}_{3} \mathrm{O}_{4}$ standard, verifying the ferrite nature of the shell. The relatively high intensity at the (400) position is attributed to the overlapping of (100) peak from the Fe core. d) CB (diameter of $16 \mathrm{~nm}$ ) had high saturation magnetization $\left(\sim 139 \mathrm{emu} \mathrm{g}^{-1}[\mathrm{Fe}]\right)$, but were superparamagnetic at $300 \mathrm{~K} . H_{e x}$, external magnetic field strength. 
a)

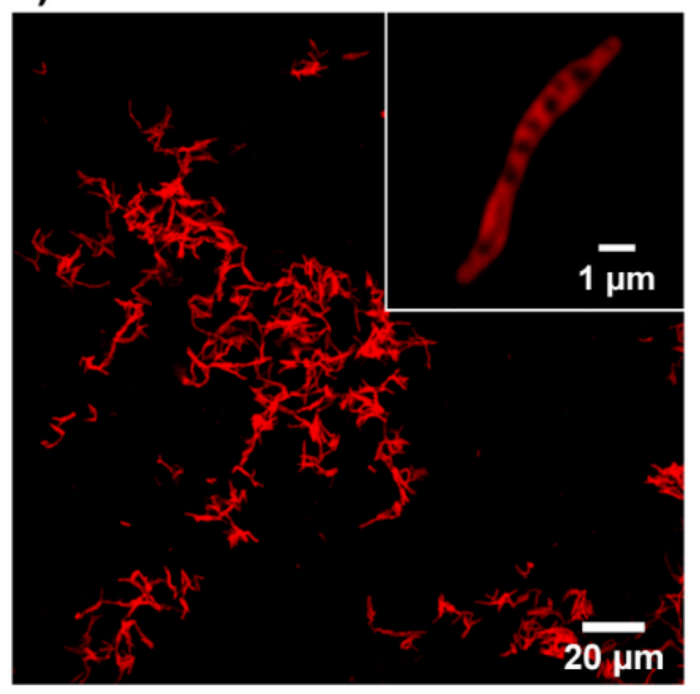

c)

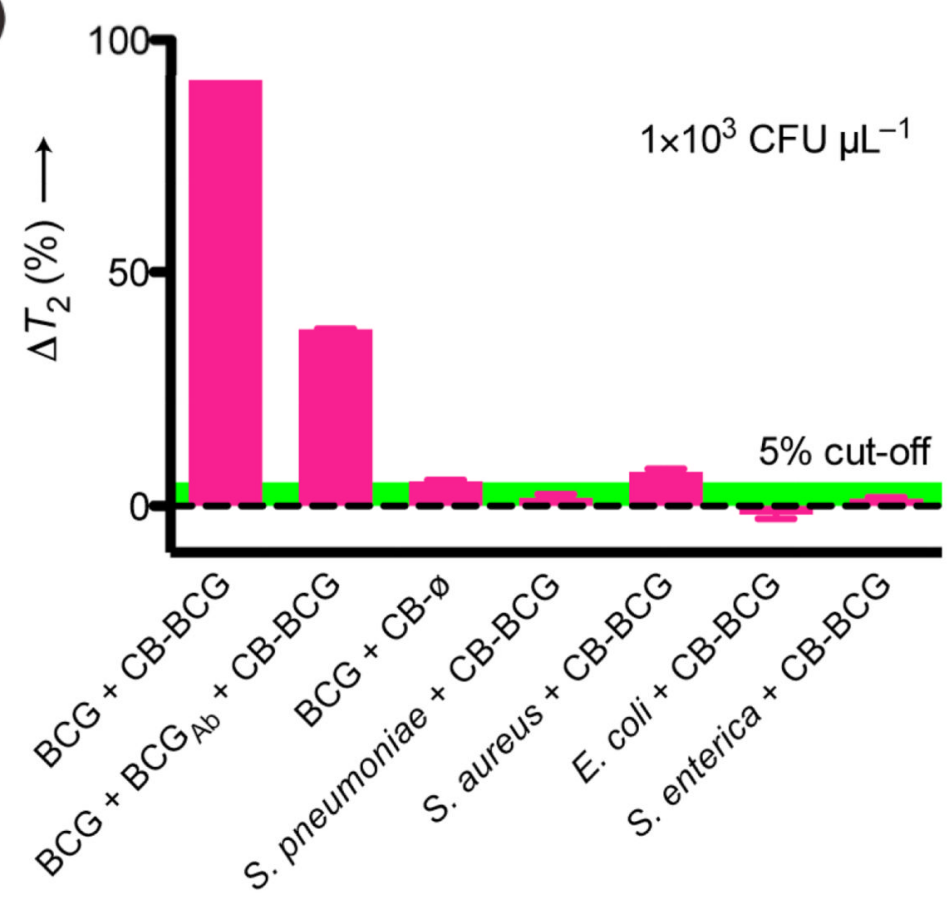

b)

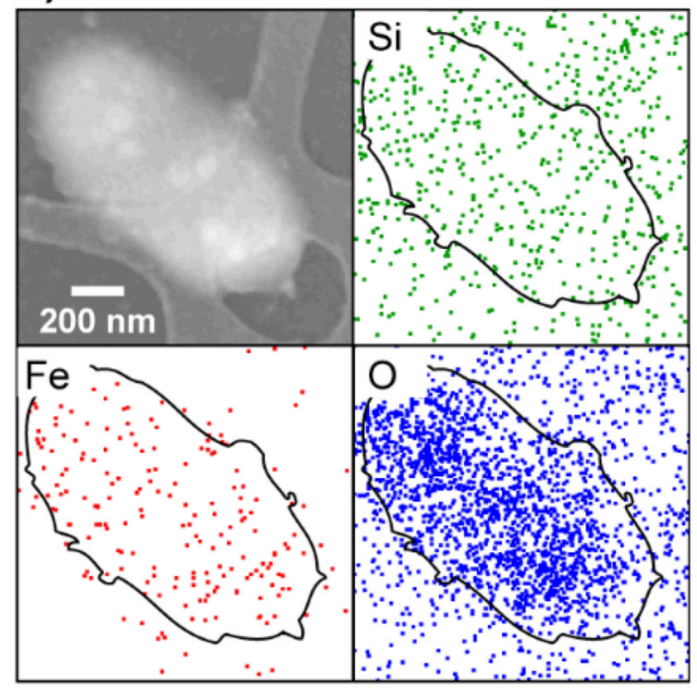

Figure 2.

Selective BCG targeting with CB-BCG. a) Confocal micrograph of BCG incubated with fluorescently labeled CB-BCG. b) Transmission electron micrograph of BCG targeted with CB-BCG. Element mapping detected high Fe signal on a whole bacterium. c) Specificity of CB-BCG. A panel of different bacteria samples were prepared as indicated $\left(\sim 10^{3} \mathrm{CFU}\right.$ $\mu \mathrm{L}^{-1}$ ) and NMR measurements were performed. The BCG sample incubated with CB-BCG showed large $T_{2}$ changes $\left(\Delta T_{2}\right)$, whereas control samples had $\Delta T_{2}<5 \%$. BCG, bacillus Calmette-Guérin; CB-BCG, CB derivatized with anti-BCG monoclonal antibody; CB- $\varnothing$, unmodified $\mathrm{CB} ; \mathrm{BCG}_{\mathrm{Ab}}$, anti-BCG monoclonal antibody. 


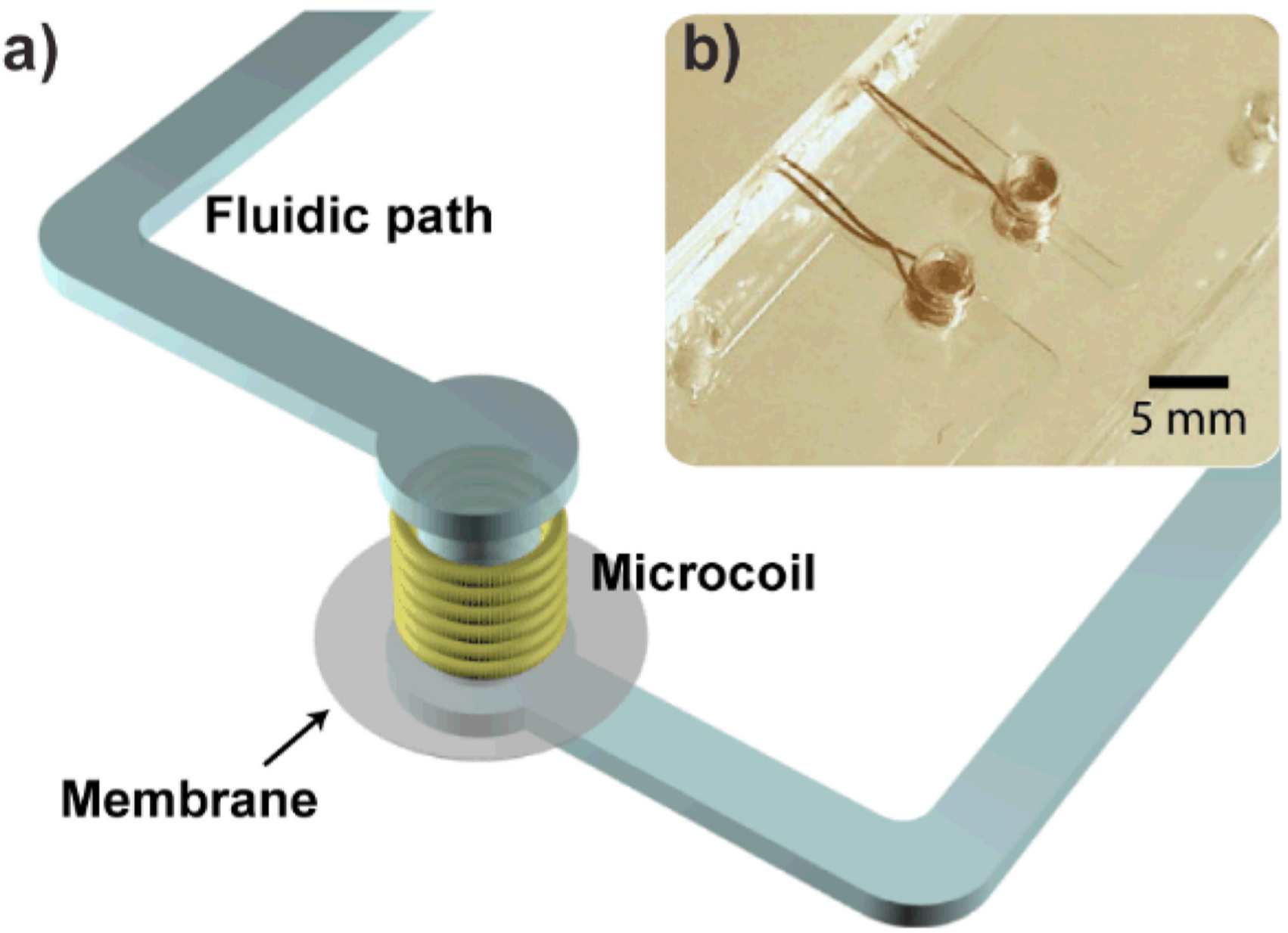

Figure 3.

NMR-filter system for bacterial concentration and detection. a) The system consists of a microcoil and a membrane filter integrated with a microfluidic channel. The microcoil is used for NMR measurements; the membrane filter concentrates bacteria inside the NMR detection chamber to achieve high detection sensitivity. b) A prototype device with two measurement sites. The NMR detection volume was $\sim 1 \mu \mathrm{L}$. 
a)
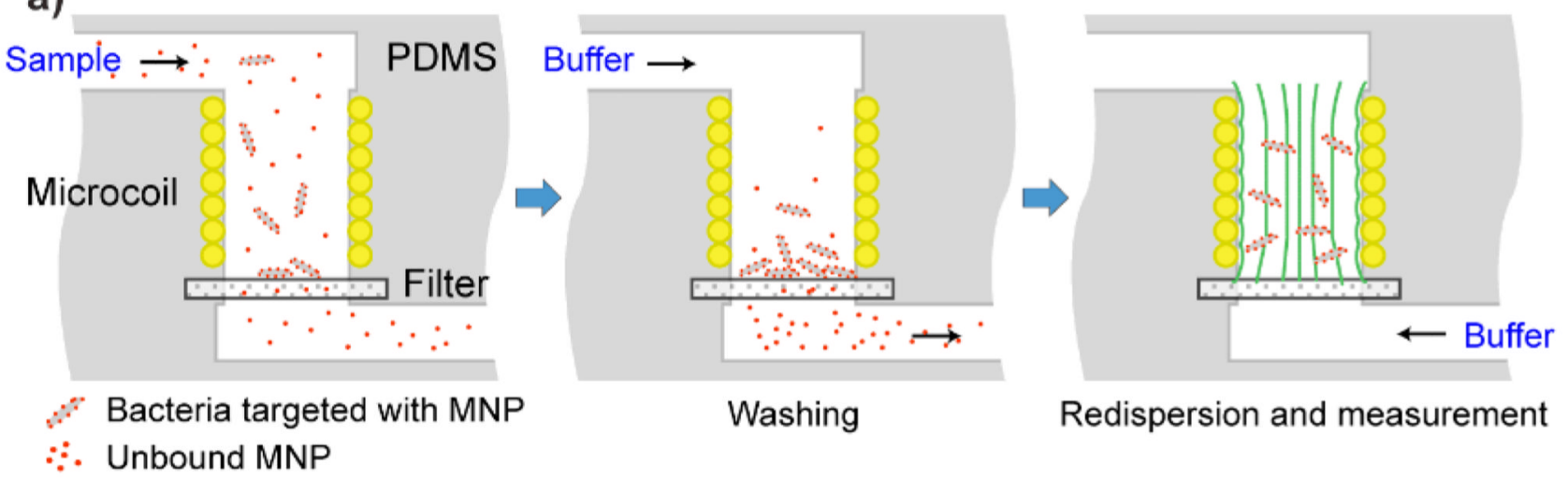

Washing

b)
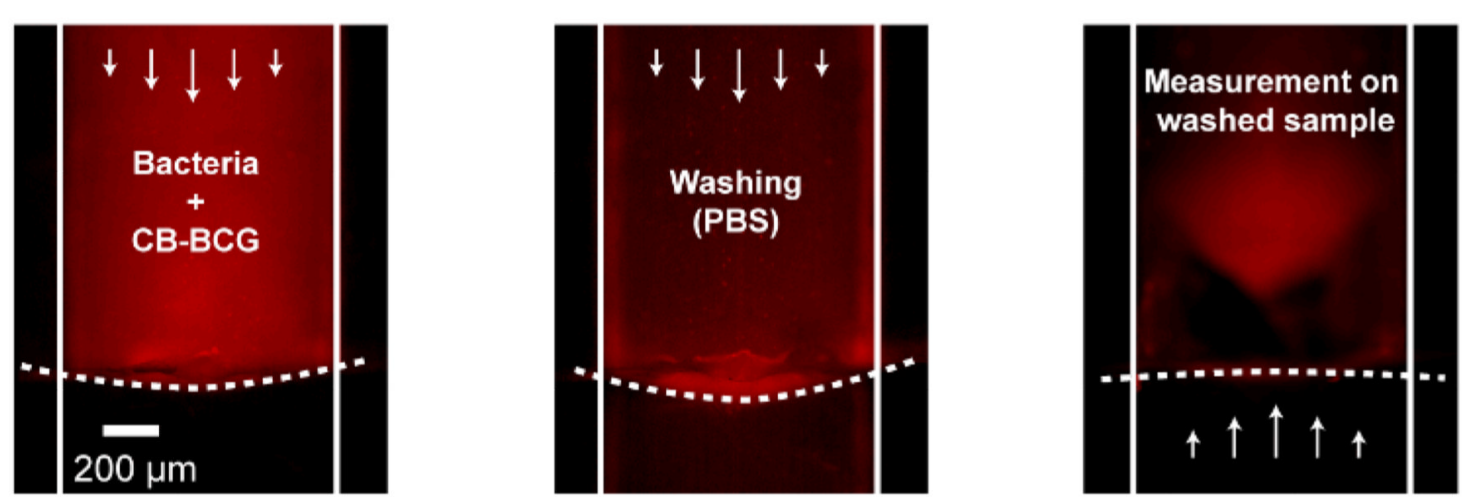

c)

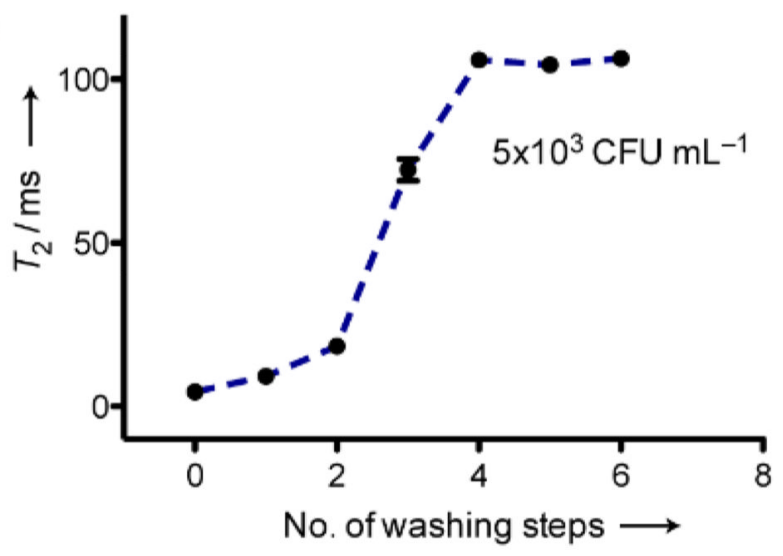

d)

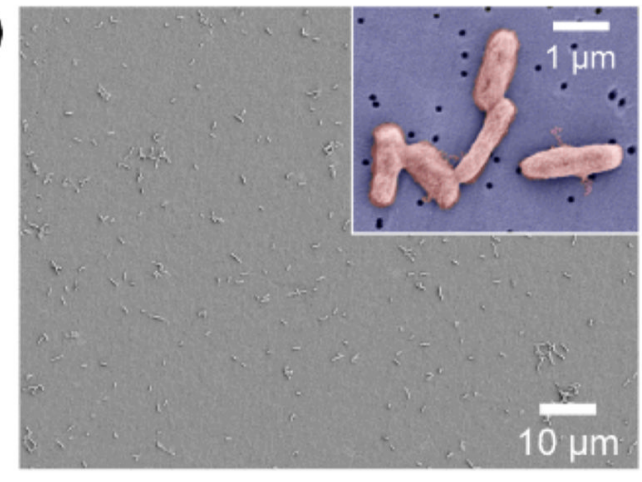

Figure 4.

Bacterial separation and concentration. a) The unprocessed sample containing bacteria and $\mathrm{CB}-\mathrm{BCG}$ is introduced into the device. The membrane filter retains bacteria while unbound CB-BCG pass through. To remove free CB-BCG, the microfluidic channel is repeatedly flushed with buffer. Finally, the flow is reversed to redisperse the captured bacteria and NMR measurements are performed. b) Demonstration of bacteria capture. A device without a microcoil was used for optical microscopy. c) The ideal number of wash steps to remove unbound CB was determined. Following each wash with PBS $(100 \mu \mathrm{L})$, the sample was redispersed and NMR measurements were performed. Note that $T_{2}$ reached a plateau after 4 
washes. d) After the final wash step, the membrane filter was imaged to confirm bacterial capture. 


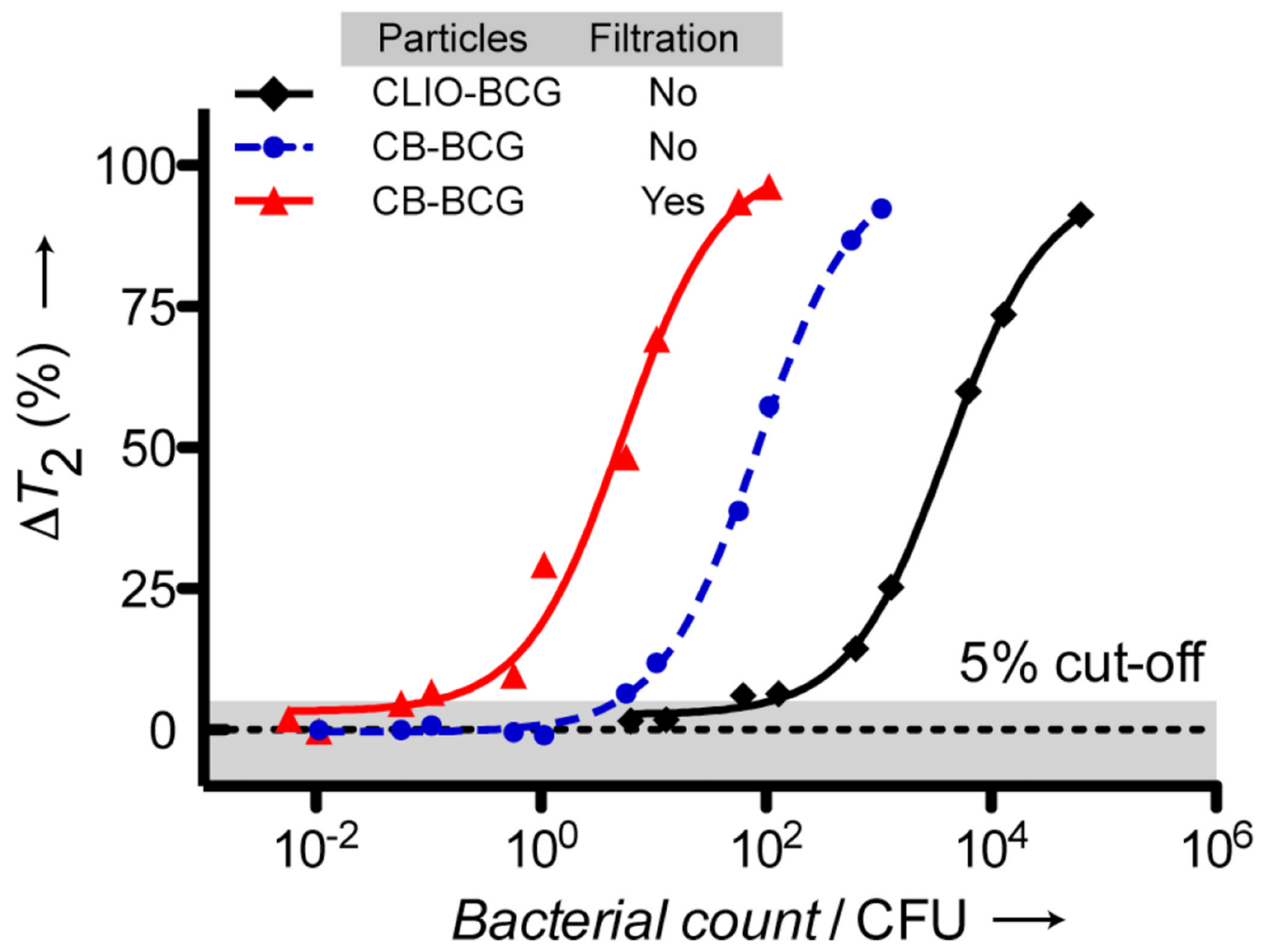

Figure 5.

Comparison of detection sensitivity. Measurements were performed on samples with varying BCG counts to determine detection sensitivities. First, a microfluidic chip without a membrane filter was used to determine the intrinsic mass-detection limits. BCG were targeted either with CB-BCG or CLIO-BCG. With CB-BCG, we already achieved a massdetection limit of $\sim 6 \mathrm{CFU}$ ( $1 \mu \mathrm{L}$ detection volume), much lower than that of $\sim 100 \mathrm{CFU}$ for CLIO-BCG. When CB-BCG-targeted samples $(100 \mu \mathrm{L})$ were filtered, the concentration

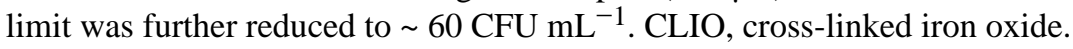

